\title{
Synthesis and antibacterial activity of certain random copolyesters from 4,4'- Oxybis(Benzoic acid)
}

\author{
N. Malathy ${ }^{1}$ and D. Roop Singh ${ }^{2^{\star}}$ \\ ${ }^{1}$ Teacher Research Fellow under University Grants Commission-Faculty Development, Presidency College, Chennai, \\ Tamil Nadu - 600005, India \\ ${ }^{2}$ Post-Graduate \& Research Department of Chemistry, Presidency College, Chennai, Tamil Nadu - 600005, India \\ n.malathymalu@yahoo.in ${ }^{1}$; roopsingh@hotmail.com²
}

\begin{abstract}
Six new random copolyesters with ethereal linkages were prepared from a potential mesogenic non-linear monomer 4, 4'-oxybis(benzoic acid) by direct poly-condensation with different aliphatic and aromatic diols in pyridine solution. Diphenylchlorophosphate (DPCP) was employed as the condensation agent. Viscosity measurements, FTIR, ${ }^{1} \mathrm{H}$ and ${ }^{13} \mathrm{C}$ NMR spectral data were used for investigating their structural features. The thermal phase transition behavior of these polymers was investigated by Differential Scanning Calorimetry (DSC). Agar well diffusion method was employed to study the antibacterial activity of these random copolyesters. All the six random copolyesters showed significant inhibition against the test bacteria. The presence of ether group in the main chain of the polyester enhances the solubility in organic solvents and hence eases processing.
\end{abstract}

Keywords: Non-linear random copolyesters, arylidene diol, antibacterial activity.

\section{Introduction}

Polymers are used as biocidial agents in recent times. By incorporating biologically active organic moieties into the polymer backbone, the activities can be introduced. In terms of their biological activity, these polymers are more effective than their monomers. Such polymers are known for their biocidial activity against some bacterial, fungal and viral strains. Cho (Sang Taek et al., 1996) prepared polymers containing diphenylether which when assayed against Staphylococcus aureus by shake flask method was reported it to be bactericidal. Therapeutic uses of a variety of drug carrier system have significant impact on the treatment and potential cure of many chronic diseases such as cancer, diabetes, arthritis and HIV infection. Polymeric systems can behave as drug-delivering agents to deliver drugs directly to the intended site of action and can also improve efficacy while minimizing unwanted side effects elsewhere in the body which often limit the long term use of the drugs.

Suhas Thattle et al. (2005) reported on the role of polymeric systems that can deliver drugs directly to the intended site of action and enhance antibacterial efficacy. Adrina Popa et al. (2003) grafted quaternary phosphonium salts on to polymer supports and found it to be bactericidal against Escherichia coli, Staphylococcus aureus and Pseudomonas aeruginosa. Hyun et al. (2006) reported on the surface characterization of 8-quinolinyl acrylate-grafted poly (ethylene terephthlate) prepared by plasma glow discharge and its antibacterial activity. Jatin Patel et al. (2006) synthesized polymethyl acrylates and investigated their antimicrobial activity against bacteria. Alexander et al. (2003) reported that textiles covalently modified with alkylated polyethylenimine are strongly bactericidal against several airborne gram-positive and gram-negative bacteria.

Over half the hospital acquired bacterial infection that arise in patients are caused by medical implants, such as catheters, prosthetics and sensors. In response to this, Mark Schoenfisch (2000) of the University of North Carolina and fellow researchers has developed polymer coatings that prevent bacteria sticking to implant surfaces. According to Schoenfisch implant-derived infections are difficult to treat because the bacteria stick to the implant, creating a thin film or 'biofilm'. Bacteria within biofilm are often resistant to conventional antibiotics. Implants coated with antibacterial polymers can resist biofilm formation, enabling them to better integrate with the patients' tissue. The polymer coatings are loaded with antimicrobial drugs. In some cases the drugs are slowly released from the polymer matrix, in others, biodegradable polymers gradually break down and free the drugs. University of Washington researchers Horbett et al. (1999) have developed a method of crafting medical implants from an antibacterial polymer that could prevent thousands of patients from dying of hospitalacquired infections each year. The polymer slowly releases an antibiotic to keep bacteria from establishing a foothold. It could be used to prevent infection around such commonly used devices as catheters as well as more permanent implants, such as pacemakers.

Recently, Rajakumar et al. (2005; 2006) synthesized dentritic architectures using 4, 4'-dihydroxy bis (arylidene) cyclopentanones and studied their antibacterial activity by disc diffusion method. Reuben Jonathan (2007) reported the antibacterial activity of certain poly (esteramides) synthesized from 4, 4'-oxybis (benzoic acid). Chitra and coworkers (2010) reported the bactericidal activity of certain copolyesters containing bischalcone moiety in the main chain.

This paper deals with the synthesis, characterization and investigation of bactericidal activity of six nonlinear random copolyesters by direct polycondensation of mesogenic 4,4'-oxybis(benzoic acid) [4, 4'-OBBA] with certain aliphatic diols and arylidene diols. All of these
Research article

CCIndian Society for Education and Environment (iSee)
"Antibacterial activity of copolyesters" http://www.indjst.org
Malathy \& Roop Singh Indian J.Sci.Technol. 
random copolyesters were characterized with a variety of experimental techniques including viscosity measurements, qualitative solubility tests and spectral studies. Agar well diffusion method (National Committee, 1993) was employed to study the antibacterial activity of random copolyesters.

\section{Materials and methods}

\section{Materials}

Pyridine (Merck, 99\% pure) used as polymerization medium, was refluxed over potassium hydroxide and distilled (B.P: $115^{\circ} \mathrm{C}$ ). Lithium chloride anhydrous (Aldrich, Analar) was dried in vacuum. Methanol (B.P. $65^{\circ} \mathrm{C}$ ) was purified by refluxing over quicklime and distilled before use. Vanillin (Aldrich, Analar), phydroxybenzaldehyde (Aldrich99\%), cyclohexanone (Aldrich) and cyclopentanone (Aldrich) were used as such in the synthesis of arylidenediols. 4,4'oxybis(benzoic)acid, 1,4-butanediol, 1,3-propanediol, 4,4'-biphenol and diphenylchlorophosphate were purchased from Aldrich Chemicals and used without further purification.

Antimicrobial studies

The agar diffusion method was followed for antibacterial susceptibility test. Petri plates were prepared by pouring $10 \mathrm{ml}$ of Muller Hinton Agar for bacteria and allowed to solidify. These agar plates were inoculated with $0.1 \mathrm{ml}$ of standardised bacterial suspension $\left(2 \times 10^{6}\right.$ cells $\left./ \mathrm{ml}\right)$ and uniformly spread. A $6 \mathrm{~mm}$ well was cut and filled with $10 \%$ DMSO of synthetic compounds. A well filled with $10 \% \mathrm{DMSO}$ served as control. The diameter of the inhibition zone observed around the well was measured for each bacterium after $48 \mathrm{hrs}$ of incubation at $37^{\circ} \mathrm{C}$. Ciprofloxacine $(5 \mu \mathrm{g} /$ disc) was used as the standard. The bacterial activity of the random copolyesters was assayed against Escherichia coli, Staphyloccus aureus, Klebsiella pneumoniae, Vibrio cholerae and Bacillus cereus.

\section{Preparation of arylidene diols}

Arylidene diols used in the synthesis of the polyesters were prepared by the condensation of the respective ketone with aromatic hydroxy aldehydes in the mole ratio 1:2. Bis (4-hydroxybenzylidene) cycloalkanones and bis (4-hydroxy3-methoxybenzylidene) cycloalkanones were synthesized by the already reported method (Kannappan et al., 2000).

Synthesis of copolysters

All the six copolyesters were prepared by direct polycondensation of two diols and one diacid (Table1) in the respective mole ratio $1: 1: 2$ in pyridine solution using diphenylchlorophosphate (DPCP) as condensation agent (Higashi et al., 1983, Sun et al., 2000). This method gives the polyesters in high yield and avoids the tedious preparation of acidchlorides.

1,4-BD: 1,4 - Butane diol

4,4' - BP: 4,4' - Biphenol

1, 3 - PR: 1, 3 - Propane diol

$\mathrm{BHCH}: \quad \mathrm{Bis}(4-h y d r o x y b e n z y l i d e n e)$ cyclohexanone
BHCP: Bis(4-hydroxybenzylidene) cyclopentanone

BVCH: Bis(4-hydroxy 3-methoxybenzylidene) cyclohexanone

4,4'-OBBA: 4,4'-oxybis(benzoic acid)

$\eta_{\text {inh }} \mathrm{dL} / \mathrm{g}$ - inherent viscosity, $\mathrm{T}_{\mathrm{g}}{ }^{\circ} \mathrm{C}$ - glass transition temperature, $\mathrm{T}_{\mathrm{m}}{ }^{\circ} \mathrm{C}$ - melting temperature

Characterization

The inherent viscosity of the co-polyesters was determined in $\mathrm{N}, \mathrm{N}$-dimethylacetamide (DMAc) solution at $30^{\circ} \mathrm{C}$ using an Ubbelohde viscometer. The solubility of these polyesters was tested in various solvents qualitatively. The polyesters reported here were soluble in solvents such as DMSO, DMAc, DMF and THF and partially soluble in n-butanol, chloroform and ethylacetate.

IR spectra of the co-polyesters were recorded using Nicolet 510 FTIR analyzer with their neat films in $\mathrm{KBr}$ pellets. The ${ }^{1} \mathrm{H}$ NMR and ${ }^{13} \mathrm{C}$ NMR spectra were recorded with JEOL GSX - $400 \mathrm{MHz}$ instrument in DMSO- $\mathrm{d}_{6} / \mathrm{CDCl}_{3}$ solvent with TMS as internal reference. In thermal analysis, DSC thermograms were recorded in Dupont 2910 differential scanning calorimeter using $5 \mathrm{mg}$ samples under nitrogen atmosphere at a heating rate of $10^{\circ} \mathrm{C} / \mathrm{min}$.

\section{Results and discussion}

All the six copolyesters synthesized from 4, 4'oxybis(benzoic acid) are more soluble. The presence of ether group in the main chain of polyester enhances the solubility in organic solvents and hence facilitates processing (Pradip et al., 1996). The percentage yield of the polyesters and their inherent viscosity values determined in $\mathrm{N}, \mathrm{N}$-dimethylacetamide (DMAc) solutions at $303 \mathrm{~K}$ are given in Table 1 . The percentage yield of the polyesters EBPR and EPPR synthesized using aliphatic diol is found to be low when compared to polyesters EBBP, EBVH, EPBH and EPVH obtained using arylidene diols. It is observed that $\eta_{\text {inh }}$ values of the polymers derived using aliphatic diols are lower than that of those derived from arylidene diols.

Spectral characterization

Fig. 1. IR spectrum of random copolyester EPVH

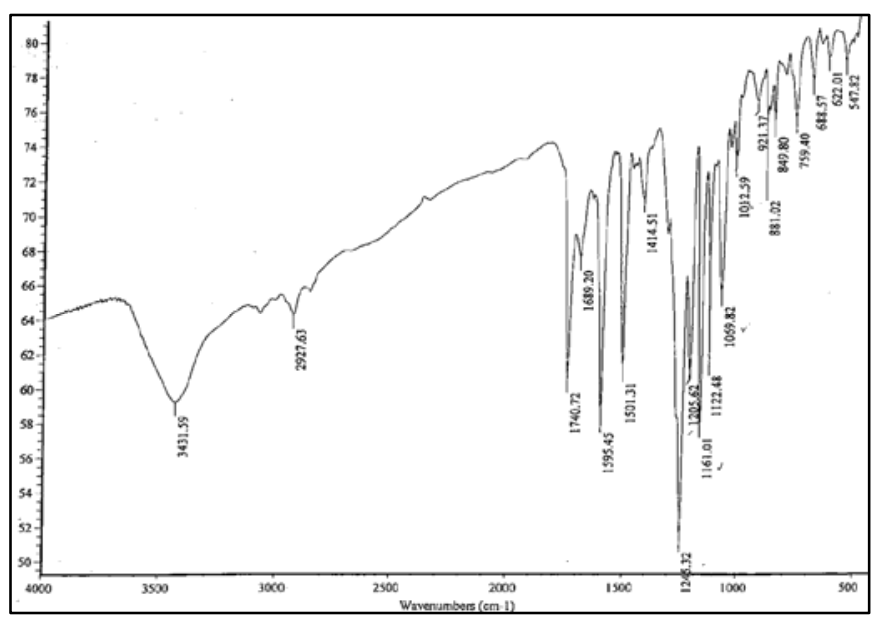


Fig. 2. ${ }^{7} H$ NMR spectrum of random copolyester EPBH

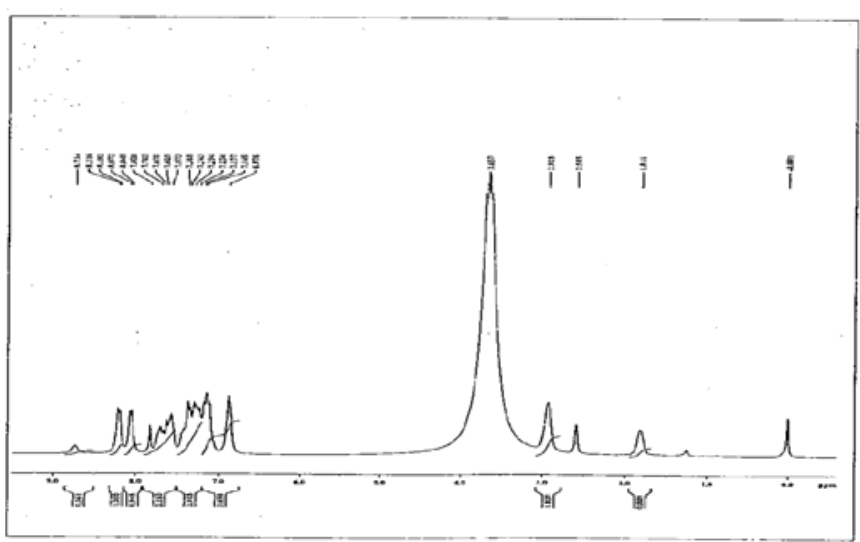

The IR spectra of all the six random copolyesters showed characteristic absorption in the range of 1710$1750 \mathrm{~cm}^{-1}$ due to ester $\mathrm{C}=\mathrm{O}$ stretching frequency. Fig. 1 shows an IR spectrum of polymer EPVH taken at room temperature. It may be noted that the absorption at $v$ $=1689 \mathrm{~cm}^{-1}$ in EPVH indicate that arylidene keto moiety is incorporated into the polymer chain. The ${ }^{1} \mathrm{H}$ NMR spectrum presented in Fig.2 indicate that the signal at $\delta=2.5-3.5 \mathrm{ppm}$ shows the presence of methylene protons of cyclohexanone of arylidene keto group of EPBH. The aromatic protons of dicarboxylic acid shows absorption at about $\delta=7.0-8.2 \mathrm{ppm}$. The microstructure of the repeat units in the polymer chain can be identified satisfactorily using ${ }^{13} \mathrm{C}$ NMR spectrum presented in Fig. 3 though the ${ }^{13} \mathrm{C}$ NMR spectra of the polymers are complex. The signals at $\delta=160-190 \mathrm{ppm}$ in the ${ }^{13} \mathrm{C}$ NMR spectrum of EPBH indicates the carbonyl carbon of the ester group as well as the arylidene keto moiety. The aromatic carbon atoms are indicated by the signals at $\delta=110-140 \mathrm{ppm}$. Thus, the proton decoupled ${ }^{13} \mathrm{C}$ spectrum of the polymers indicates that the polymer chain contains ester group. The copolymerization effect of these polyesters was attributed to their random placements along the polyester chain, which was also verified with ${ }^{13} \mathrm{C}$ NMR spectroscopy (Fig.3).

\section{Fig. 3. ${ }^{13} \mathrm{C}$ NMR spectrum of random copolyester EPBH}

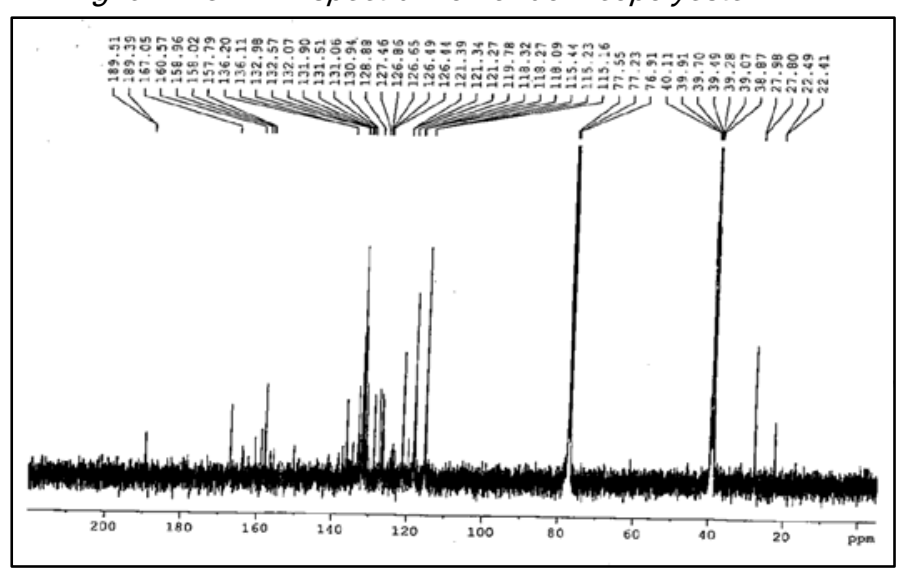

Vol. 5 No. 3 (Mar 2012)

ISSN: 0974- 6846

The thermal transition temperatures of the random copolyesters were determined from DSC thermograms (Fig. 4) and are listed in Table 1. It is observed that the $T_{g}$ of the polyesters EBBP and EBVH obtained using arylidenediols is higher than that of polyester synthesized using aliphatic diols. This may be due to the rigidity of arylidene moiety present in the polymer chain. It is also observed that the $T_{g}$ value of vanillin based polyester EPVH is higher than that of EPBH when 4, 4'-biphenol is used as the common diol, which could be due to interlocking effect of the methoxy substituent present in the arylidene keto moiety by which the methoxy substituent hooked closely to the polymer chain. There are reports on such interlocking effects on the thermal properties of polymers by Lenz and coworkers (Lenz, 1985). They suggested that the interlocking effect depends on the size of the substituent. This was further supported by reported work of Kannappan and coworkers (Kannappan et al., 2000; 2001) by ultrasonic method. The same correlation is observed for $\eta_{\text {inh }}$ values also.

Fig. 4. DSC Thermograms of random copolyesters

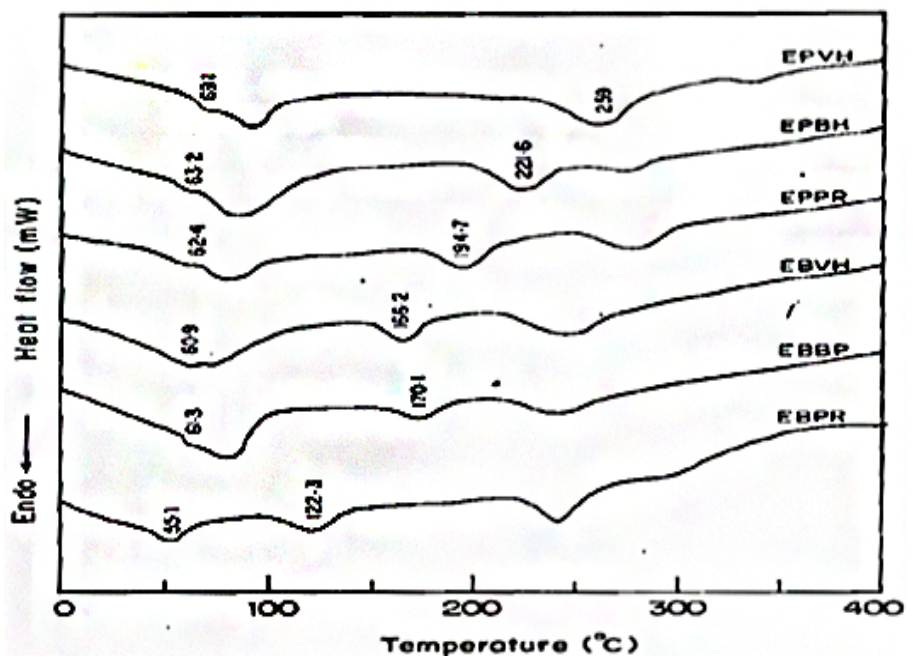

Antibacterial studies

Polymeric materials are known for their antimicrobial activities. In the present work, six polyesters have been synthesized from aliphatic diols and arylidene diols and agar well diffusion method was employed to study their antibacterial activity (Fig.5). All the six test samples were tested at different concentration to test their efficacy in inhibiting the growth of the human pathogens. The bacterial activity of EBPR, EBBP, EBVH, EPPR, EPBH and EPVH was assayed against Escherichia coli, Staphyloccus aureus, Klebsiella pneumoniae, Vibrio cholerae and Bacillus cereus. The diameters for the zone of inhibitions at different concentration against the test bacteria are given in Table 2. The standard antibiotic disc (ciprofloxacine disc $5 \mu \mathrm{g} /$ disc) inhibited the growth of Escherichia coli by $20 \mathrm{~mm}$, Staphyloccus aureus by 19 $\mathrm{mm}$, Klebsiella pneumoniae by $18 \mathrm{~mm}$, Vibrio cholerae by $19 \mathrm{~mm}$ and Bacillus cereus by $20 \mathrm{~mm}$.
Thermal characterization

Research article

CIndian Society for Education and Environment (iSee)
"Antibacterial activity of copolyesters" http://www.indjst.org
Malathy \& Roop Singh Indian J.Sci.Technol. 
Table 1. Polymer code and properties of random copolyesters

\begin{tabular}{|c|c|c|c|c|c|c|c|c|}
\hline $\begin{array}{c}\text { S. } \\
\text { No }\end{array}$ & Diol - I & Diol - II & Diacid & $\begin{array}{c}\text { Polymer } \\
\text { Code }\end{array}$ & Yield $\%$ & $\eta_{\text {inh }} \mathrm{dL} / \mathrm{g}$ & $\mathrm{T}_{\mathrm{g}}{ }^{\circ} \mathrm{C}$ & $\mathrm{T}_{\mathrm{m}}{ }^{\circ} \mathrm{C}$ \\
\hline 1. & $1,4-\mathrm{BD}$ & $1,3-\mathrm{PR}$ & $4,4^{\prime}-\mathrm{OBBA}$ & EBPR & 60 & 0.42 & 55.1 & 122.3 \\
\hline 2 & $1,4-\mathrm{BD}$ & BHCP & $4,4^{\prime}-\mathrm{OBBA}$ & EBBP & 71 & 0.63 & 61.3 & 170.1 \\
\hline 3 & $1,4-\mathrm{BD}$ & BVCH & $4,4^{\prime}-\mathrm{OBBA}$ & EBVH & 70 & 0.61 & 60.9 & 166.2 \\
\hline 4 & $4,4-\mathrm{BP}$ & $1,3-\mathrm{PR}$ & $4,4^{\prime}-\mathrm{OBBA}$ & EPPR & 66 & 0.53 & 62.4 & 194.7 \\
\hline 5 & $4,4-\mathrm{BP}$ & BHCH & $4,4^{\prime}-\mathrm{OBBA}$ & EPBH & 80 & 0.65 & 63.2 & 221.6 \\
\hline 6 & $4,4-\mathrm{BP}$ & BVCH & $4,4^{\prime}-\mathrm{OBBA}$ & EPVH & 78 & 0.68 & 69.1 & 259.0 \\
\hline
\end{tabular}

Fig.5. Antibacterial activity of random copolyester EBVH

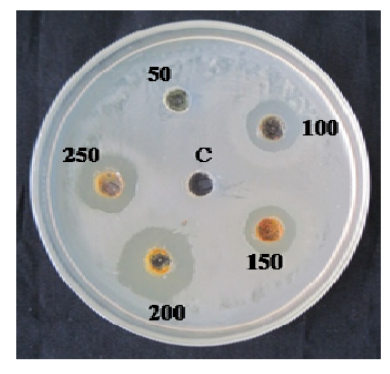

Excharichiscoof

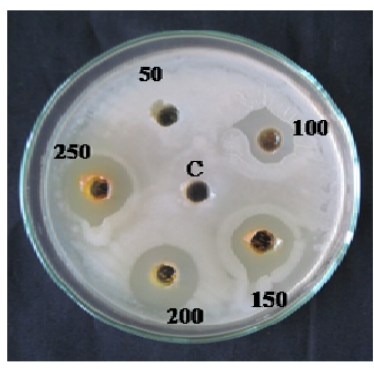

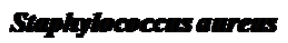

III
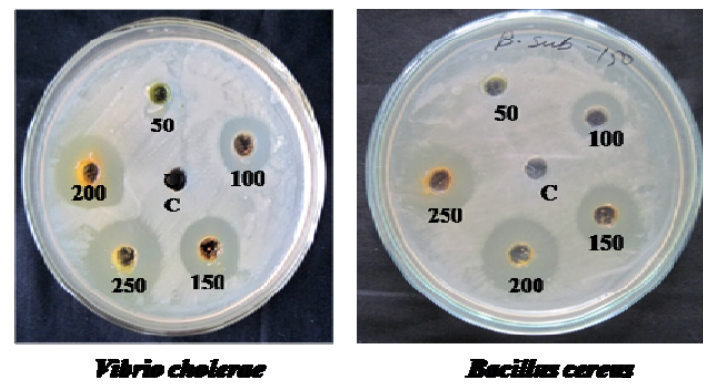

The antibacterial activity of all the random copolyesters derived from 4,4'-oxybis(benzoicacid) is high, which could be due to the presence of ether linkage in the oxybis(phenylene) moiety. Among them, higher activity was observed in vanillin based polyesters EBVH and EPVH than polyesters EBBP, EPBH obtained from 4-hydroxybenzaldehyde based arylidene diols, which may be due to the presence of methoxy group in vanillin (Rajakumar et al., 2006). The aliphatic diols exhibited antimicrobial activity (Sinskey et al., 1977) and it is interesting to note that polyesters EBPR and EPPR synthesized using aliphatic diols also exhibited higher antibacterial activity. These studies revealed that polyesters synthesized from 4,4'-OBBA using certain

\section{Acknowledgment}

aliphatic and arylidene diols exhibited antibacterial activity towards pathogenic bacteria. This also indicates that the polymer chain containing ether linkage enhances the antibacterial activity. Moreover, the antibacterial activity of random copolyesters synthesized from $4,4^{\prime}$ oxybis(benzoicacid) in this work is found to be higher than that of poly(esteramides) synthesized from 4,4'-oxybis(benzoicacid) reported by Reuben Jonathan (Reuben Jonathan, 2007).

\section{Conclusion}

All the six random copolyesters contain structurally different repeating unit. The aim of this study was to prepare random copolyesters and its antibacterial potential was evaluated with pathogenic bacteria. The results of present antimicrobial assay revealed that the random copolyesters showed good inhibitory activity against all the tested pathogens, suggesting that the presence of ether group enhances the antibacterial activity.

The author N.Malathy is thankful to the University Grants Commission of India for permitting and supporting research by Faculty Development Programme.

\section{References}

1. Adriana Popa, Davidescu C, Trif R, Illia Gh, Smaranda Illescu and Dehelean Gh (2003) Study of quaternary 'onium' salts grafted on polymers; antibacterial activity of quaternary phosphonium salts grafted on 4 gel-type styrene-divinylbenzene copolymers. React. \& Funct. Polym. 55, 151-158.

2. Alexander M Klibanov, Jian Lin, Shuyi Qui and Kim Lewis (2003) The mechanism of bactericidal and fungicidal activities of textiles covalently modified with

Table 2. Inhibition effects of certain random copolyesters on the growth of pathogenic bacteria

\begin{tabular}{|c|c|c|c|c|c|c|c|c|c|c|c|c|c|c|c|}
\hline \multirow{4}{*}{$\begin{array}{c}\text { Test } \\
\text { material }\end{array}$} & \multicolumn{3}{|c|}{ E.coli } & \multicolumn{3}{|c|}{ S.aureus } & \multicolumn{3}{|c|}{ K.pneumoniae } & \multicolumn{3}{|c|}{ V. cholerae } & \multicolumn{3}{|c|}{ B.cereus } \\
\hline & \multicolumn{15}{|c|}{ Concentration $-\mu q / \mathrm{ml}$} \\
\hline & 50 & 100 & 150 & 50 & 100 & 150 & 50 & 100 & 150 & 50 & 100 & 150 & 50 & 100 & 150 \\
\hline & \multicolumn{15}{|c|}{ Zone of inhibition in diameter $(\mathrm{mm})$} \\
\hline EBPR & 8.4 & 13.1 & 16.2 & 8.7 & 13.2 & 15.8 & 7.9 & 12.9 & 16.1 & 7.9 & 14.3 & 17.4 & 7.1 & 13.5 & 16.9 \\
\hline EBBP & 7.9 & 11.8 & 15.6 & 8.3 & 14.1 & 16.1 & 8.2 & 13.2 & 16.3 & 5.9 & 8.4 & 11.9 & 5.3 & 8.1 & 11.5 \\
\hline EBVH & 9.8 & 14.7 & 18.6 & 10.2 & 14.4 & 17.5 & 9.9 & 15.4 & 19.4 & 8.6 & 14.4 & 17.9 & 9.3 & 14.6 & 18.4 \\
\hline EPPR & 8.1 & 13.1 & 16.9 & 8.9 & 13.1 & 16.2 & 7.1 & 10.7 & 14.6 & 6.1 & 9.1 & 11.9 & 5.4 & 8.3 & 11.5 \\
\hline EPBH & 9.3 & 13.9 & 17.1 & 9.1 & 13.8 & 16.9 & \begin{tabular}{|l|}
8.2 \\
\end{tabular} & 14.1 & 17.2 & 6.4 & 13.9 & 16.7 & 6.2 & 14.2 & 17.3 \\
\hline EPVH & 8.9 & 14.1 & 17.3 & 9.8 & 13.9 & 17.4 & \begin{tabular}{|l|}
8.4 \\
\end{tabular} & 14.3 & 17.9 & 8.6 & 14.9 & 18.2 & 8.4 & 14.7 & 17.6 \\
\hline
\end{tabular}


alkylated polyethylenimine. Biotechnol. Bioeng. 83, 168-172.

3. Chitra M, Rajendran TV, Duraipandiyan V, Rajan CY and Ruben Jonathan D (2010) A study on the synthesis and bactericidal activity of certain copolyesters containing bischalcone moiety in the main chain. Indian J. Sci. \& Technol. 3, 890-893.

4. Higashi F, Hoshia A and KiyOshige J (1983) Preparation of aromatic polyesters the direct polycondensation reaction with diphenyl chlorophosphate in pyridine. J. Polym. Sci. Chem. Ed. 21, 3241-3247.

5. Horbett Thomas, Ratner and Marek W Urban (1999) Antibacterical implants could prevent Infections, save patients' lives. Sci. Daily, December, 15.

6. Jatin N Patel, Mitul B. Dolia, Mitesh B Patel and Rajni $M$ Patel (2006) 4-Chloro-3- methyl Phenyl methacrylate copolymers with 2,4dichlorophenylmethacrylate: Synthesis, characterization and antimicrobial activity. J. Appl. Polym. Sci. 100, 439-448.

7. Kannappan V, Arumugasamy E, Ravichandran E and Baskar B (2000) Synthesis and Characterization of certain thermotropic liquid crystalline random copolyesters. J. Polym. Mater. 17, 4-9.

8. Kannappan V, Mahendran S, Sathyamoorthi P and Roop Singh D (2001) Ultrasonic method of determination of glass transition temperatures of certain thermotropic liquid crystalline copolyesters. J. Poly. Mater. 18, 409-416.

9. Lenz RW (1985) Characterization of thermotropic liquid crystalline polymers. J. Pure \& Appl. Chem. 57, 977-984.

10. Mark A Schoenfisch, Marc Ovadia and Jeanne E Pemberton (2000) Covalent surface chemical modification of electrodes for cardiac pacing applications. J. Biomed. Material Res. 51, 209-215.

11. National Committee for Clinical Laboratory Standards (1993) Methods for dilution in antimicrobial susceptibility tests. Approved Standard M2 - A5. NCCLS, Villanova, PA.

12. Pradip K Bhowmik, Edward DT, Atkins Robert W Lenz and HaeooskHan (1996) Crystalline and liquid crystalline polyesters of phenyl-substituted 4, 4'Biphenols. 5. Copolymers with 4,4'-Oxybis(benzoic acid). Macromol. 29, 3778-3786.

13. Rajakumar P, Ganesan K, Jayavelu $S$ and Murugesan K (2005) Synthesis and bactericidal efficacy of novel dendrimers. Syn. Lett. 7, 1121-1124.

14. Rajakumar P, Ganesan K, Jayavelu $S$ and Murugesan K (2006) Synthesis and Antibacterial activity of dendritic architectures. Synthesis. 3, 528532.

15. Reuben Jonathan D (2007) Synthesis, characterization and antibacterial activity of certain Poly (ester-amides), Univ. Madras. pp: 101.
Vol. 5 No. 3 (Mar 2012)

ISSN: 0974- 6846

16. Sang Taek Oh, Sun Hong Han, Chang Sik Ha and Won Jei Cho (1996) Polymers as antimicrobial peptide mimics. J. Appl. Polym. Sci. 59, 1871-1878.

17. Seok-Hee Hyun, Min-Woo Kim, Dae-Hee Oh, JnnKyu Kang and Woo-Sik Kim (2006) Surface Characterization of 8-quinolinyl acrylate-grafted poly(ethylene terephthalate) prepared by plasma glow discharge and its antibacterial activity. J. Appl. Polym. Sci. 101, 863-868.

18. Sinskey AJ, Akedo M and Gomez R (1977) Antimicrobial action of aliphatic diols and their Esters. J. Food Sci. 3, 699-701.

19. Suhas Thattle, Kapil Datar and Raphael M Ottenbrite (2005) Perspectives on polymeric drugs and drug delivery systems. J. Bioact. Compt. Polym. 20, 585601.

20. Sun SJ, Liao YC and Chang JC (2000) Studies on the synthesis and properties thermotropic liquid crystalline polycarbonates. VII. Liquid crystalline polycarbonates and poly (ester-carbonates) derived from various mesogenic groups. J. Polym. Sci. Part A: Polym. Chem. 38, 1852-1860. 